${ }^{1}$ Programa de PósGraduação em Políticas Públicas, Centro de Ciências Humanas e Letras, Universidade Federal do Piauí. Campus Universitário Ministro Petrônio Portela, Ininga. 64049-550 Teresina PI Brasil.

jorgeomaia@hotmail.com

\section{Pagamento por desempenho em sistemas e serviços de saúde: uma revisão das melhores evidências disponíveis}

\author{
Pay-for-performance in health care services: \\ a review of the best evidence available
}

Abstract Pay-for-performance (P4P) has been widely used around the world seeking to improve health outcomes, and in Brazil it is the basis of the National Program for Improving Access and Quality (PMAQ). The literature published between 1998 and January 2013 that evaluated the effectiveness of $P 4 P$ to produce results or patterns of access and quality in health was scrutinized. A total of 138 studies, with the inclusion of a further 41 studies (14 systematic reviews, 07 clinical trials and 20 observational studies) were retrieved and analyzed Among the more rigorous studies, favorable conclusions for P4P were less frequent, whereas observational studies were more favorable to positive effects of P4P on the quality of, and access to, health services. Methodological limitations of observational studies may have contributed to these results, but the range of results is more linked to the conceptual and contextual aspects of the use of the P4P schemes reviewed, the heterogeneity of P4P models and results. P4P can be helpful in promoting the achievement of objectives in health care systems, especially in the short term and for specific actions requiring less effort of health care providers, but should be used with caution and with a rigorous planning model, also considering undesirable or adverse effects.

Key words Pay-for-performance, Program for Improving Access and Quality (PMAQ)
Resumo O Pagamento por Desempenho (P4P) é usado em todo o mundo visando à melhoria dos resultados em saúde, e no Brasil é base do Programa Nacional de Melhoria do Acesso e da Qualidade (PMAQ), lançado pelo Ministério da Saúde, em 2011. Revisou-se a literatura publicada entre 1998 e janeiro de 2013, sobre a efetividade do P4P, para produzir resultados ou padrões de acesso e qualidade na saúde. Foram recuperados e analisados 138 estudos, sendo incluídos 41 (14 revisões sistemáticas, 7 ensaios clínicos e 20 estudos observacionais). Estudos mais rigorosos foram menos favoráveis ao P4P, enquanto estudos observacionais apontaram efeitos positivos do P4P sobre a qualidade e o acesso nos serviços de saúde. Limitações metodológicas dos estudos observacionais podem ter contribuído para tais resultados, mas a variedade de resultados está mais ligada aos aspectos conceituais e contextuais dos esquemas de P4P avaliados, reforçando a heterogeneidade de modelos e resultados do P4P. O P4P pode ser útil para promover o alcance de objetivos em sistemas de saúde, especialmente no curto prazo e para ações pontuais que exijam menos esforço dos provedores de serviços de saúde, mas deve ser utilizado com cautela e com planejamento rigoroso do modelo, considerando-se também efeitos indesejáveis ou adversos.

Palavras-chave Pagamento por desempenho, Programa Nacional de Melhoria do Acesso e da Qualidade (PMAQ) 


\section{Introdução}

O pagamento por desempenho (pay-for-performance, $\mathrm{P} 4 \mathrm{P})$, ou financiamento baseado em resultados (result-based-financing, RFB), consiste na transferência de dinheiro, a provedores ou serviços de saúde, condicionada a resultados alcançados em face de ações ou metas mensuráveis e predeterminadas ${ }^{1-4}$. Embora muitos modelos já tenham sido identificados, esquemas de P4P na saúde em geral objetivam incentivar condutas individuais ou coletivas para a obtenção de melhores resultados ou de padrões de qualidade na provisão de serviços de saúde ${ }^{5,6}$.

Em âmbito internacional, o P4P é defendido e utilizado para melhorar a qualidade do atendimento e alcançar metas institucionais nas políticas de saúde ${ }^{1,2}$. Apesar do P4P ser uma das estratégias dominantes na busca da melhoria da qualidade dos sistemas e organizações de saúde ${ }^{7}$, ainda persiste considerável lacuna nas evidências sobre sua efetividade ${ }^{1,5}$.

No Brasil, o Ministério da Saúde lançou, em 2011, o Programa Nacional de Melhoria do Acesso e da Qualidade da Atenção Básica (PMAQ), com o objetivo de induzir a ampliação da capacidade da gestão tripartite (federal, estadual e municipal) do Sistema Único de Saúde (SUS) e ampliação da oferta e qualidade dos serviços de Atenção Primária à Saúde (APS), em face das necessidades de saúde da população. O PMAQ instituiu recursos financeiros adicionais para os municípios participantes, os quais estão condicionados ao alcance de padrões de acesso e qualidade, e se operacionaliza em ciclos de adesão/ contratualização, desenvolvimento e avaliação externa. A conclusão do primeiro ciclo de avaliação do PMAQ se deu em agosto de 2012, incluindo mais de 16 mil equipes de atenção básica, em 3700 municípios $^{7}$, sendo esta iniciativa especialmente focada na estratégia Saúde da Família, modelo predominante de APS no SUS 8,9 .

No campo da investigação sobre os efeitos do P4P, ainda são pouco disponíveis estudos realizados no Brasil, mas no âmbito internacional, Oxman e Fretheim ${ }^{1}$ identificaram 12 revisões sistemáticas que avaliaram algum aspecto dos efeitos do P4P no setor de saúde, considerando pacientes, prestadores de serviços individuais, equipes de saúde, organizações e governos. Os resultados apontaram que incentivos financeiros para provedores individuais de saúde, podem ser eficazes em curto prazo, desde que reflitam sobre metas comportamentais bem definidas, distintas e simples, mas os efeitos do P4P para mudanças complexas e de longo prazo nos processos de trabalho ainda precisam ser mais estudados.

A depender do modelo adotado, o P4P também pode produzir efeitos inesperados ou indesejáveis, motivando comportamentos distorcidos (ignorar importantes tarefas não recompensadas), 'mapismo' (fraude na apresentação de relatórios), 'escolha a dedo' (seleção de pacientes com base na facilidade para atingir metas), aumento da lacuna de recursos entre os ricos e os pobres (disparidades motivadas por determinantes sociais) e dependência dos incentivos financeiros ${ }^{1}$.

Mannion e Davies ${ }^{5}$ identificaram as seguintes características presentes nos diversos modelos de P4P por eles analisados: i) objetivos e metas utilizados; ii) unidades de avaliação; iii) medidas de desempenho; iv) análise e interpretação dos dados; e v) tamanho da recompensa. Os objetivos e metas, assim como as unidades de avaliação e medidas de desempenho podem abranger uma gama de variáveis (volume de atendimentos, satisfação e segurança do usuário, custo-efetividade dos tratamentos, dentre outras possibilidades). Os incentivos financeiros podem concentrar-se em indivíduos, equipes ou instituições e o tamanho da recompensa, fator mais relevante segundo os autores, pode variar de muito pequena, produzindo efeitos insignificantes, a grandes valores, os quais podem levar a mudanças rápidas e potencialmente imprevisíveis, aumentando, inclusive, os riscos de consequências adversas ${ }^{5}$.

Diante da gama de incertezas relacionadas ao $\mathrm{P} 4 \mathrm{P}$, uma abordagem abrangente da literatura publicada sobre a efetividade do uso de esquemas de pagamento por desempenho para obter padrões de acesso e qualidade em serviços e sistemas de saúde, pode subsidiar a discussão e a tomada de decisão sobre a temática, que hoje integra a agenda da política nacional de saúde, notadamente no âmbito da atenção básica. Assim, o objetivo desse estudo de revisão da literatura foi identificar, avaliar e sistematizar as evidências disponíveis sobre a efetividade do P4P na saúde.

\section{Métodos}

Esta revisão da literatura guiou-se pela questão -problema da efetividade do $\mathrm{P} 4 \mathrm{P}$ em produzir resultados ou padrões de acesso e qualidade em serviços de saúde. Buscou-se identificar estudos publicados de 1998 até janeiro de 2013, incluindo-se apenas revisões sistemáticas (com ou sem metanálise), ensaios clínicos controlados e estudos observacionais, do tipo caso-controle, coorte 
e cross-section, que avaliaram a eficácia/efetividade do P4P para melhorar ou padronizar processos/resultados do âmbito da provisão de serviços de saúde, utilizando-se incentivos financeiros individuais, para equipes de saúde ou organizações/instituições.

Uma busca estruturada foi feita nas bases indexadas da Biblioteca Virtual em Saúde (BVS) e PubMed entre 20 de dezembro de 2012 e 20 de janeiro de 2013, utilizando-se a seguinte estratégia de sensibilização: "pay for performance" OR "payfor-performance" OR "paying for performance" OR "financial incentives" OR "reimbursement, incentive" OR "incentive reimbursement". Também foram utilizados ainda os seguintes limites para a filtragem dos artigos de interesse, a fim de refinar os resultados iniciais da busca: > Assunto principal: Reembolso de incentivo; > Idiomas: Inglês/espanhol/português; > Ano de publicação: 2000/2012 > Tipo de estudo: Revisões Sistemáticas; Ensaios Clínicos Controlados; Estudos de Caso-Controle; Estudos de Coorte.

Para a avaliação da qualidade da evidência, utilizou-se o modelo para avaliação da qualidade de revisões sistemáticas, adaptado de Guyatt et al. ${ }^{10}$, pelas diretrizes metodológicas para elaboração de pareceres técnico-científicos do Ministério da Saúde ${ }^{11}$. Para os ensaios clínicos e estudos observacionais, a qualidade foi avaliada seguindo-se as recomendações das diretrizes metodológicas para a elaboração de revisões sistemáticas e metanálise de ensaios clínicos randomizados do Ministério da Saúde ${ }^{12}$, bem como os critérios de identificação de limitações metodológicas que resultem em viés ou erro aleatório nos resultados dos estudos, conforme preconizado por Guyatt et al. ${ }^{10}$.

Para decidir sobre o nível de recomendação da evidência, considerou-se que, dentre os estudos incluídos e após a análise crítica do desenho metodológico no atendimento aos critérios básicos de sua caracterização $0^{10-12}$, as revisões sistemáticas gozaram do nível de recomendação mais elevado, seguidas dos ensaios clínicos controlados e dos estudos observacionais (casocontrole, coorte e cross-section). Após a leitura e categorização dos artigos, os achados foram sistematizados e dispostos de forma sintética, destacando-se os objetivos e as principais conclusões, bem como o grau de recomendação da evidência sobre a efetividade do uso do $\mathrm{P} 4 \mathrm{P}$ para melhorar ou padronizar processos/resultados do âmbito da provisão de serviços de saúde.

\section{Resultados}

O pagamento por desempenho tem recebido atenção crescente da pesquisa em sistemas de saúde (Health Systems Research) e sido amplamente defendido como estratégia/ferramenta para promover mudanças comportamentais em provedores de serviços de saúde favoráveis a melhores resultados, tanto em nível individual como coletivo $^{1-3}$. No entanto, os artigos incluídos nesta revisão apresentaram resultados contraditórios quanto à efetividade do $\mathrm{P} 4 \mathrm{P}$ para melhorar ou obter padrões de qualidade no âmbito de serviços e sistemas de saúde utilizando incentivos financeiros baseados em resultados, a depender dos diferentes modelos conceituais, objetivos e formas de implantação.

A busca sensibilizada recuperou 138 estudos, dos quais, após análise crítica, foram incluídos 41 artigos, dos quais 14 configuraram revisões sistemáticas, 07 atenderam ao desenho de ensaios clínicos e 20 relataram estudos observacionais, conforme descrito no Quadro 1.

\section{Revisões sistemáticas \\ sobre os efeitos do P4P na saúde}

Revisões sistemáticas diferem das revisões de literatura tradicionais por incluir apenas resultados de estudos primários de larga escala que atendam ao desenho metodológico de ensaios clínicos controlados e, quando possível, também aleatorizados, que abordem uma questão clínica claramente formulada, utilizando métodos sistemáticos e explícitos para identificar, selecionar e avaliar criticamente estudos que se enquadrem nos critérios metodológicos pré-definidos, extrair e analisar seus dados e sintetizá-los de forma clara e objetiva. Assim, as revisões sistemáticas são amplamente aceitas como as mais confiáveis fontes de evidências sobre os efeitos das intervenções de saúde e cada vez mais são usadas para identificar, avaliar e combinar evidências sobre as consequências das intervenções, considerando aspectos clínicos e econômicos, dado que sua abordagem sistemática e explícita reduz o risco de vieses e facilita a avaliação crítica dessas sínteses ${ }^{13}$.

Dentre as 37 revisões recuperadas na etapa inicial de busca, 14 estudos foram incluídos ${ }^{14-27}$, por atenderem ao problema proposto de investigar a efetividade do P4P para produzir melhorias nos resultados em saúde e possuírem qualidade 
metodológica adequada, segundo os parâmetros escolhidos nesta avaliação ${ }^{10,11}$. Destas, 13 constituíram revisões sistemáticas de estudos primários $^{14-25,27}$ (ensaios clínicos controlados, randomizados ou não) e uma revisão de revisões sistemáticas sobre os efeitos do P4P também foi considerada $^{28}$, tendo em vista a qualidade metodológica verificada nessa síntese.

As revisões sistemáticas apresentaram conclusões conservadoras acerca das evidências sobre os efeitos benéficos do $\mathrm{P} 4 \mathrm{P}$ sobre os resultados em saúde, em parte devido ao reconhecimento da baixa qualidade metodológica comumente encontrada entre os estudos primários disponíveis sobre os efeitos do $\mathrm{P} 4 \mathrm{P}$, apontada em vários dos estudos ${ }^{14-18,22-27}$, em parte porque os autores defendem que vários aspectos do $\mathrm{P} 4 \mathrm{P}$ ainda carecem de mais investigação, como, por exemplo, os riscos de produzir comportamentos e resultados adversos ou indesejáveis.

A melhoria de indicadores isolados, especialmente aqueles de entrega e cobertura de serviços e ações de saúde pontuais, parece ser mais facilmente alcançada pelo $\mathrm{P} 4 \mathrm{P}$, ao passo que os benefícios do uso do P4P para mudanças complexas e de longo prazo que incluem resultados em nível sistêmico não puderam ser completamente evidenciados ${ }^{14-18,22-27}$. As maiores lacunas de evidências sobre o P4P se referem aos custos e relações daí decorrentes (custo-benefício, custo -efetividade e custo-utilidade) e aos riscos potenciais envolvidos no seu uso nos diversos níveis de agente-alvo do incentivo financeiro, considerando diferentes contextos organizacionais.

Algumas conclusões encontradas nos estudos incluídos, entretanto, podem ser usadas como

Quadro 1. Resultados da busca de artigos nas bases eletrônicas (PubMed e BVS).

\begin{tabular}{|c|c|c|c|c|}
\hline Bases & $\begin{array}{l}\text { Busca realizada em 20/12/12 } \\
\text { (atualizada em 20/01/13) }\end{array}$ & Recuperados & Selecionados & Incluídos \\
\hline 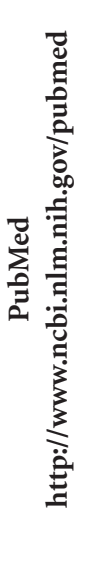 & $\begin{array}{l}\text { Termos: } \\
\text { "pay for performance" OR "pay-for- } \\
\text { performance" OR "paying for performance" } \\
\text { OR “financial incentives" OR "reimbursement, } \\
\text { incentive” OR “incentive reimbursement" } \\
\text { Limites ativados: } \\
\text { > Article types: Clinical Trial; Controlled } \\
\text { Clinical Trial; Meta-Analysis; Randomized } \\
\text { Controlled Trial; Systematic Reviews; >Text } \\
\text { availability: Abstract available; Free full text } \\
\text { available; Full text available; > Publication } \\
\text { dates: From 2000/01/01 to 2012/12/31; } \\
\text { > Species: Humans; > Languages: English; } \\
\text { Spanish; Portuguese. }\end{array}$ & $\begin{array}{l}\text { Revisões da } \\
\text { literatura } \\
(27) \\
\text { Ensaios Clínicos } \\
\text { em geral } \\
(57) \\
\text { Estudos } \\
\text { Observacionais } \\
(20) \\
\text { Total } \\
(104)\end{array}$ & $\begin{array}{l}\text { Revisões } \\
\text { Sistemáticas } \\
(06) \\
\text { Ensaios Clínicos } \\
\text { Controlados } \\
(04) \\
\text { Estudos } \\
\text { Observacionais } \\
(10) \\
\text { Total } \\
(20)\end{array}$ & $\begin{array}{l}\text { Revisões } \\
\text { Sistemáticas } \\
(04) \\
\text { Ensaios Clínicos } \\
\text { Controlados } \\
(03) \\
\text { Estudos } \\
\text { Observacionais } \\
(10) \\
\text { Total } \\
(17)\end{array}$ \\
\hline 范 & $\begin{array}{l}\text { Termos: } \\
\text { "pay for performance" OR "pay-for- } \\
\text { performance" OR “paying for performance” } \\
\text { OR “financial incentives" OR “reimbursement, } \\
\text { incentive” OR “incentive reimbursement" } \\
\text { Limites ativados: } \\
\text { > Assunto principal: Reembolso de incentivo; } \\
\text { > Idiomas: Inglês/espanhol/português; > } \\
\text { Ano de publicação: 2000/2012; > Tipo de } \\
\text { estudo: Revisões Sistemáticas; Ensaios Clínicos } \\
\text { Controlados; Estudos de Caso-Controle; } \\
\text { Estudos de Coorte. }\end{array}$ & $\begin{array}{l}\text { Revisões } \\
\text { Sistemáticas } \\
(10) \\
\text { Ensaios Clínicos } \\
\text { Controlados } \\
(14) \\
\text { Estudos } \\
\text { Observacionais } \\
(12) \\
\text { Total } \\
(34)\end{array}$ & $\begin{array}{l}\text { Revisões } \\
\text { Sistemáticas } \\
(10) \\
\text { Ensaios Clínicos } \\
\text { Controlados } \\
(08) \\
\text { Estudos } \\
\text { Observacionais } \\
(10) \\
\text { Total } \\
(28)\end{array}$ & $\begin{array}{l}\text { Revisões } \\
\text { Sistemáticas } \\
(10) \\
\text { Ensaios Clínicos } \\
\text { Controlados } \\
(04) \\
\text { Estudos } \\
\text { Observacionais } \\
(10) \\
\text { Total } \\
(24)\end{array}$ \\
\hline
\end{tabular}

Fonte: elaboração própria. 
subsídios na averiguação e discussão dos potenciais resultados da utilização do $\mathrm{P} 4 \mathrm{P}$ em sistemas de saúde, especialmente quanto aos seus elementos constitutivos e respectivos efeitos daí decorrentes. De forma geral, os efeitos do P4P dependeriam do modelo usado, incluindo o tipo de intervenção proposta, tamanho e frequência dos incentivos, quais as metas e objetivos buscados, como são mensurados os resultados para o pagamento dos incentivos, bem como de outros componentes institucionais, tais como a existência de suporte técnico para o programa e o contexto organizacional em que o mesmo é implantado, além de fatores de confusão de natureza socioeconômica, por exemplo. O Quadro 2 apresenta as principais conclusões das revisões sistemáticas.

Em síntese, a parte considerável dos estudos concluiu pela insuficiência de evidências que suportem o uso do P4P para promover melhorias da qualidade e resultados em saúde que dependam de intervenções e mudanças complexas e de longo prazo, como, por exemplo, na redução das disparidades em saúde entre grupos étnicos e socioeconômicos, ou para melhorar de forma sistêmica os resultados dos cuidados primários em saúde. Noutro lado, ainda há incertezas sobre os riscos e custos envolvidos na utilização do $\mathrm{P} 4 \mathrm{P}$ na saúde, sendo possível que efeitos indesejáveis ocorram, tais como desídia dos provedores individuais com tarefas que não são recompensadas, fraude de informações, aprofundamento das disparidades em saúde e dependência financeira dos incentivos ${ }^{15,18,26}$.

Entretanto, a efetividade do $\mathrm{P} 4 \mathrm{P}$ como estratégia para mediar mudanças pontuais e de curto prazo também foi apontada por alguns dos estudos, especialmente para ampliar a cobertura de serviços ou condutas específicas, tais como ações de imunização de grupos etários por vacinação, adesão a protocolos clínicos e terapêuticos e rastreamento (screening) de doenças específicas (câncer, por exemplo) $)^{13,17,18,26}$.

Por fim, os estudos de revisão sistemática sobre os efeitos do P4P sobre medidas de desempenho e resultados em saúde, reclamam a disponibilidade de mais estudos primários com boa qualidade metodológica, que proporcionem evidências mais robustas sobre as consequências do uso do P4P na saúde ${ }^{14-27}$.

\section{Ensaios Clínicos Controlados sobre os efeitos do P4P na saúde}

Ensaios clínicos controlados são estudos experimentais que visam ao conhecimento dos efeitos de intervenções em saúde, especialmente para o tratamento e diagnósticos de doenças, e consistem numa ferramenta poderosa para a obtenção de evidências para a prática clínica ${ }^{10}$. Ensaios clínicos controlados também têm sido utilizados para avaliar os efeitos de intervenções em sistemas de saúde, como demonstram os resultados da busca de artigos que relataram estudos que avaliaram os efeitos do $\mathrm{P} 4 \mathrm{P}$ no âmbito de serviços de saúde e adotaram esse desenho metodológico.

Inicialmente, identificaram-se 71 estudos, dos quais 12 foram selecionados para leitura completa e 07 incluídos no escopo desta revisão ${ }^{29-35}$. Os resultados dos ensaios clínicos analisados apresentaram resultados variados acerca da efetividade do P4P, a depender do modelo e do contexto de implantação dos incentivos. Objetivos pontuais, envolvendo melhoria das coberturas de serviços ou adesão a condutas específicas parecem ser mais facilmente afetados pelo P4P, replicando aspecto já verificado nos resultados das revisões sistemáticas avaliadas nesse estudo.

Os estudos que abordaram ações pontuais identificaram resultados mais conclusivos sobre a efetividade do $\mathrm{P}^{2} \mathrm{P}^{29-31,34}$, sendo a intervenção associada a melhorias nas coberturas e adesão dos provedores de serviços a ações específicas em pelo menos três estudos ${ }^{30,32,34}$. Por outro lado, pelo menos dois dos estudos favoráveis ao $\mathrm{P} 4 \mathrm{P}$ indicaram que a melhoria observada foi marginal ${ }^{29-35}$, e outros dois estudos não verificaram diferenças significativas entre os grupos de intervenção e de controle ${ }^{30,32}$. Entretanto, pelo menos um dos estudos incluídos apontou resultados muito favoráveis do uso do P4P para melhoria do acesso e qualidade de um serviço de saúde materno-infantil em nível sistêmico ${ }^{33}$, sendo esse resultado ainda mais instigante por abordar os efeitos da intervenção no contexto socioeconômico de extrema carência da África subsaariana. No Quadro 3 são apresentados os principais achados dos estudos incluídos.

Assim, reforça-se a perspectiva de que os efeitos do $\mathrm{P} 4 \mathrm{P}$ são condicionados pelos elementos constitutivos dos modelos de incentivo e por fatores contextuais, que incluem elementos do âmbito institucional e do contexto socioeconômico onde a intervenção foi implantada. Nenhum dos estudos avaliou potenciais riscos ou a ocorrência de efeitos adversos/indesejáveis advindos do uso do P4P. 
Quadro 2. Síntese dos resultados da revisão da literatura (Revisões Sistemáticas).

\begin{tabular}{|c|c|c|c|}
\hline \multicolumn{4}{|c|}{ Revisões Sistemáticas (14 estudos incluídos) } \\
\hline Estudo & Objetivo & Principais resultados de interesse para o $\mathrm{P} 4 \mathrm{P}$ & Período \\
\hline Stone et al. ${ }^{14}$ & $\begin{array}{l}\text { Avaliar a eficácia de } \\
\text { diferentes abordagens } \\
\text { para melhorar a } \\
\text { imunização contra } \\
\text { influenza e pneumonia } \\
\text { bacteriana e o } \\
\text { screening de câncer } \\
\text { (cólon, mama e útero). }\end{array}$ & $\begin{array}{l}\text { Incentivos financeiros para os provedores não } \\
\text { melhoraram significativamente a utilização de serviços } \\
\text { de imunização. }\end{array}$ & Até 1999 \\
\hline $\begin{array}{l}\text { Chaix- } \\
\text { Couturier et } \\
\text { al. }{ }^{15}\end{array}$ & $\begin{array}{l}\text { Identificar modelos } \\
\text { de P4P endereçados } \\
\text { aos profissionais de } \\
\text { saúde e avaliar seus } \\
\text { efeitos sobre custos, } \\
\text { processos de trabalho e } \\
\text { resultados em saúde. }\end{array}$ & $\begin{array}{l}\text { Vários tipos de incentivos financeiros, fatores de } \\
\text { confusão e riscos foram identificados. Os fatores de } \\
\text { confusão incluíram: idade do clínico; experiência } \\
\text { e qualificação; lugar e tipo de prática médica; } \\
\text { existência de sanções por excesso de prescrição; tipo } \\
\text { e gravidade da doença e tipo de seguro-saúde. Os } \\
\text { riscos identificados foram: limitação do acesso a certos } \\
\text { tipos de cuidados, descontinuidade do tratamento e } \\
\text { ocorrência de conflitos de interesse entre o médico e } \\
\text { o paciente. O uso de incentivos financeiros reduziu } \\
\text { as prescrições em até } 24 \% \text { e os dias de hospitalização } \\
\text { em até } 80 \% \text {. Incentivos financeiros podem reduzir } \\
\text { o uso de recursos da saúde, melhorar a adesão a } \\
\text { diretrizes clínicas ou atingir metas gerais de saúde, } \\
\text { mas, considerando a baixa qualidade metodológica dos } \\
\text { estudos incluídos na revisão, a validade externa dessas } \\
\text { conclusões é limitada. }\end{array}$ & $\begin{array}{l}\text { Janeiro } \\
\text { de } 1993 \text { a } \\
\text { maio de } \\
1999\end{array}$ \\
\hline $\begin{array}{l}\text { Petersen et } \\
\text { al. }^{16}\end{array}$ & $\begin{array}{l}\text { Avaliar os efeitos do } \\
\text { P4P para melhorar } \\
\text { medidas de qualidade } \\
\text { na saúde. }\end{array}$ & $\begin{array}{l}\text { A revisão sugere que incentivos financeiros } \\
\text { endereçados a provedores individuais, grupos de } \\
\text { provedores e sistemas de saúde podem ter efeitos } \\
\text { positivos. O monitoramento e a avaliação contínuos de } \\
\text { programas de incentivo são necessários para identificar } \\
\text { eventuais efeitos indesejados sobre a qualidade do } \\
\text { atendimento. }\end{array}$ & $\begin{array}{l}\text { Janeiro } \\
\text { de } 1980 \text { a } \\
\text { novembro } \\
\text { de } 2005\end{array}$ \\
\hline $\begin{array}{l}\text { Giuffrida et } \\
\text { al. }{ }^{17}\end{array}$ & $\begin{array}{l}\text { Avaliar o impacto do } \\
\text { P4P sobre a prática } \\
\text { médica e os resultados } \\
\text { na Atenção Primária à } \\
\text { Saúde. }\end{array}$ & $\begin{array}{l}\text { O uso de da remuneração por metas na APS foi } \\
\text { associado a melhorias nas taxas de imunização, mas } \\
\text { os estudos incluídos na revisão não tiveram qualidade } \\
\text { suficiente para responder se a remuneração por metas } \\
\text { representa uma estratégia efetiva para melhorar os } \\
\text { cuidados de saúde primários. }\end{array}$ & $\begin{array}{l}1966 \mathrm{a} \\
1997\end{array}$ \\
\hline Sturm et al. ${ }^{18}$ & $\begin{array}{l}\text { Determinar os } \\
\text { efeitos do P4P sobre } \\
\text { resultados e custos de } \\
\text { saúde relacionados à } \\
\text { prescrição e uso de } \\
\text { medicamentos. }\end{array}$ & $\begin{array}{l}\text { Incentivos financeiros podem limitar despesas com } \\
\text { medicamentos, restringindo o volume de prescrições } \\
\text { ou aumentando o uso de medicamentos genéricos. } \\
\text { Como a maioria dos estudos incluídos apresentaram } \\
\text { sérias limitações metodológicas, os resultados devem } \\
\text { ser interpretados com cautela. }\end{array}$ & $\begin{array}{l}2003 a \\
2005\end{array}$ \\
\hline
\end{tabular}


Quadro 2. continuação

\begin{tabular}{|c|c|c|c|}
\hline \multicolumn{4}{|c|}{ Revisões Sistemáticas (14 estudos incluídos) } \\
\hline Estudo & Objetivo & Principais resultados de interesse para o $\mathrm{P} 4 \mathrm{P}$ & Período \\
\hline Witter et al. ${ }^{19}$ & $\begin{array}{l}\text { Avaliar as evidências } \\
\text { sobre os efeitos do } \\
\text { P4P na provisão de } \\
\text { cuidados e resultados } \\
\text { de saúde em países de } \\
\text { baixa e média renda. }\end{array}$ & $\begin{array}{l}\text { É fraca a base de evidências dos efeitos do P4P sobre } \\
\text { a prestação de cuidados e resultados em saúde. O } \\
\text { financiamento baseado no desempenho não é uma } \\
\text { intervenção uniforme, mas consiste numa série de } \\
\text { abordagens diferenciadas. Os efeitos dependem da } \\
\text { interação de diversas variáveis, incluindo o desenho } \\
\text { da intervenção, a magnitude dos incentivos, as metas e } \\
\text { como elas são medidas, o montante de financiamento } \\
\text { adicional, além de outros componentes auxiliares, tais } \\
\text { como o suporte técnico e fatores contextuais, incluindo } \\
\text { o organizacional em que é implementado. }\end{array}$ & Até 2011 \\
\hline Scott et al..$^{20}$ & $\begin{array}{l}\text { Avaliar os efeitos das } \\
\text { mudanças no modelo } \\
\text { de remuneração } \\
\text { sobre a qualidade dos } \\
\text { cuidados na Atenção } \\
\text { Primária à Saúde. }\end{array}$ & $\begin{array}{l}\text { O uso de incentivos financeiros para melhorar a } \\
\text { qualidade da APS está crescendo. No entanto, ainda } \\
\text { não há provas suficientes para apoiar seu uso para } \\
\text { melhorar a qualidade dos cuidados de saúde. O P4P } \\
\text { deve ser usado com cautela e esquemas de incentivos } \\
\text { devem ser cuidadosamente estudados antes da } \\
\text { implementação. }\end{array}$ & $\begin{array}{l}2000 \mathrm{a} \\
2010\end{array}$ \\
\hline $\begin{array}{l}\text { Van Herck et } \\
\text { al. }{ }^{21}\end{array}$ & $\begin{array}{l}\text { Avaliar os efeitos do } \\
\text { P4P e o impacto do } \\
\text { design do esquema de } \\
\text { incentivos, bem como } \\
\text { identificar mediadores } \\
\text { contextuais que afetam } \\
\text { esses efeitos. }\end{array}$ & $\begin{array}{l}\text { Evidências sobre o impacto do P4P na coordenação, } \\
\text { continuidade, centralidade no paciente e custo- } \\
\text { efetividade são limitadas. O P4P funciona como } \\
\text { estímulo a depender do objetivo pretendido: apoiar } \\
\text { padrões mínimos de qualidade e/ou a melhoria } \\
\text { desta. Os efeitos variaram de acordo com escolhas } \\
\text { de concepção e características do contexto de } \\
\text { implementação. O P4P deve usar metas de melhoria } \\
\text { a partir de linhas de base, indicadores de resultados } \\
\text { como medidas-alvo; envolver as partes interessadas; } \\
\text { comunicar informações de forma completa e explícita; } \\
\text { visar tanto a qualidade como a realização e distribuir } \\
\text { incentivos em nível individual e/ou da equipe. } \\
\text { Os resultados verificados variaram de ausentes a } \\
\text { fortemente benéficos, mas foram condicionados às } \\
\text { opções de modelo e contexto de implementação. }\end{array}$ & $\begin{array}{l}1990 \text { a } \\
\text { julho de } \\
2009\end{array}$ \\
\hline Lee et al. ${ }^{22}$ & $\begin{array}{l}\text { Avaliar os efeitos do } \\
\text { P4P sobre as atitudes } \\
\text { gerais dos prestadores } \\
\text { de serviços em saúde. }\end{array}$ & $\begin{array}{l}\text { Profissionais de saúde ainda têm um baixo nível de } \\
\text { consciência sobre P4P e podem preferir não participar } \\
\text { deste tipo de esquema. Indicadores de qualidade } \\
\text { adequados e apoio adicional para a implementação do } \\
\text { P4P se fazem necessários, na visão dos prestadores de } \\
\text { cuidados em saúde. A implementação bem sucedida do } \\
\text { P4P pode estar condicionada ao aumento do nível dos } \\
\text { conhecimentos dos prestadores de serviços sobre P4P, } \\
\text { apoio técnico e educacional, desenvolvimento de uma } \\
\text { relação de cooperação com os provedores individuais e } \\
\text { uso de medidas de qualidade mais precisas. }\end{array}$ & $\begin{array}{l}\text { Até } \\
\text { dezembro } \\
\text { de } 2011\end{array}$ \\
\hline
\end{tabular}


Quadro 2. continuação

\begin{tabular}{|c|c|c|c|}
\hline \multicolumn{4}{|c|}{ Revisões Sistemáticas (14 estudos incluídos) } \\
\hline Estudo & Objetivo & Principais resultados de interesse para o $\mathrm{P} 4 \mathrm{P}$ & Período \\
\hline $\begin{array}{l}\text { de Bruin et } \\
\text { al. }{ }^{23}\end{array}$ & $\begin{array}{l}\text { Analisar os efeitos do } \\
\text { P4P sobre a qualidade } \\
\text { e os custos da provisão } \\
\text { de cuidados e gestão } \\
\text { das doenças crônicas. }\end{array}$ & $\begin{array}{l}\text { Os incentivos financeiros mais encontrados foram } \\
\text { recompensas seletivas concedidas com base no } \\
\text { desempenho absoluto. Informações sobre a motivação, } \\
\text { certeza, tamanho, frequência e duração dos incentivos } \\
\text { financeiros foram muito limitadas. A maioria dos } \\
\text { estudos incluídos na revisão mostrou algum efeito } \\
\text { positivo do P4P sobre a qualidade de saúde, mas } \\
\text { sua qualidade metodológica impõe limitações à } \\
\text { interpretação desses efeitos. Não foram encontrados } \\
\text { estudos que avaliaram os efeitos da P4P sobre os } \\
\text { custos em saúde. As evidências sobre os efeitos do } \\
\text { uso de esquemas de P4P para incentivar a melhoria } \\
\text { da qualidade da prestação de cuidados e gestão das } \\
\text { doenças crônicas são limitadas. }\end{array}$ & $\begin{array}{l}2000 a \\
2010\end{array}$ \\
\hline $\begin{array}{l}\text { Eldridge e } \\
\text { Palmer }^{24}\end{array}$ & $\begin{array}{l}\text { Avaliar o uso do } \mathrm{P} 4 \mathrm{P} \\
\text { em sistemas de saúde } \\
\text { nos países de baixa } \\
\text { renda. }\end{array}$ & $\begin{array}{l}\text { Ainda há pouco consenso na literatura sobre o } \\
\text { significado ou o conceito de pagamento baseado } \\
\text { em desempenho. Estudos multidisciplinares são } \\
\text { necessários para investigar as vantagens e desvantagens } \\
\text { do P4P. Questões relativas à adaptação do P4P a } \\
\text { sistemas de saúde menos desenvolvidos ainda precisam } \\
\text { ser resolvidas. }\end{array}$ & $\begin{array}{l}1990 \mathrm{a} \\
2008\end{array}$ \\
\hline Gillam et al. ${ }^{25}$ & $\begin{array}{l}\text { Analisar as evidências } \\
\text { dos impactos do } \\
\text { Quality and Outcomes } \\
\text { Framework (mais } \\
\text { amplo programa de } \\
\text { P4P da Inglaterra). }\end{array}$ & $\begin{array}{l}\text { As melhorias na qualidade do atendimento de doenças } \\
\text { crônicas foram modestas no âmbito do Quality and } \\
\text { Outcomes Framework inglês e o impacto sobre os } \\
\text { custos, postura profissional e experiência do paciente } \\
\text { ainda permanecem incertos. Organizações de saúde } \\
\text { devem manter a cautela sobre os potenciais benefícios } \\
\text { de regimes semelhantes. }\end{array}$ & $\begin{array}{l}\text { Janeiro } \\
\text { de } 2004 \text { a } \\
\text { julho de } \\
2011\end{array}$ \\
\hline $\begin{array}{l}\text { Oxman e } \\
\text { Fretheim }{ }^{26}\end{array}$ & $\begin{array}{l}\text { Avaliar a efetividade } \\
\text { do P4P para atingir } \\
\text { metas de saúde. } \\
\text { (Revisão de revisões } \\
\text { sistemáticas). }\end{array}$ & $\begin{array}{l}\text { O P4P pode ser eficaz no curto prazo e para metas } \\
\text { simples e distintas sobre comportamentos bem } \\
\text { definidos. Não há evidências de que o P4P sustente } \\
\text { mudanças de longo prazo. Os esquemas de P4P } \\
\text { também podem produzir efeitos indesejáveis, tais } \\
\text { como comportamentos não intencionais, distorções } \\
\text { (ignorando as tarefas que não são recompensadas), } \\
\text { fraude, ampliação das disparidades em saúde e } \\
\text { dependência dos incentivos financeiros. Evidências da } \\
\text { efetividade do P4P para melhorar resultados em saúde } \\
\text { são limitadas e quase nenhuma evidência do custo- } \\
\text { benefício do P4P está disponível. }\end{array}$ & $\begin{array}{l}\text { Até agosto } \\
\text { de } 2007\end{array}$ \\
\hline $\begin{array}{l}\text { Emmert et } \\
\text { al. }^{27}\end{array}$ & $\begin{array}{l}\text { Analisar a literatura } \\
\text { existente sobre } \\
\text { avaliação econômica } \\
\text { do P4P. }\end{array}$ & $\begin{array}{l}\text { Com base em avaliações econômicas completas } \\
\text { a eficiência do P4P não pode ser demonstrada. } \\
\text { Avaliações econômicas parciais mostraram resultados } \\
\text { mistos, mas falhas metodológicas limitam sua } \\
\text { interpretação. Os resultados mostraram que a } \\
\text { evidência da eficácia do P4P é escassa e inconclusiva. } \\
\text { Faz-se necessária mais investigação abordando a } \\
\text { eficiência do P4P. }\end{array}$ & $\begin{array}{l}\text { Janeiro } \\
\text { de } 2000 \\
\text { a abril de } \\
2010\end{array}$ \\
\hline
\end{tabular}

Fonte: Elaboração própria. 
Quadro 3. Síntese dos resultados da revisão da literatura (Ensaios Clínicos Controlados).

\begin{tabular}{|c|c|c|c|}
\hline \multicolumn{4}{|c|}{ Ensaios Clínicos Controlados (7 estudos incluídos) } \\
\hline Estudo & Objetivos do estudo & Principais resultados de interesse para o $\mathrm{P} 4 \mathrm{P}$ & Período \\
\hline $\begin{array}{l}\text { Kouides } \\
\text { RW et } \\
\text { al. }^{29}\end{array}$ & $\begin{array}{l}\text { Investigar o efeito do P4P sobre a taxa } \\
\text { de vacinação contra influenza, em } \\
\text { idosos. }\end{array}$ & $\begin{array}{l}\text { No grupo de médicos que recebeu o incentivo, a taxa } \\
\text { de imunização média foi de 68,6\% (DP 16,6\%), em } \\
\text { comparação com 62,7\% (DP 18,0\%) no grupo de } \\
\text { controle }(\mathrm{P}=0,22) \text {. A melhoria na média das coberturas } \\
\text { de imunização foi 10,3\% no grupo de incentivo em } \\
\text { comparação com 3,5\% no grupo controle }(\mathrm{P}=0,03) \text {. } \\
\text { O incentivo financeiro foi responsável pelo aumento } \\
\text { de aproximadamente 7\% na cobertura de imunização } \\
\text { contra influenza de idosos. }\end{array}$ & 1991 \\
\hline $\begin{array}{l}\text { Hillman } \\
\text { et al. }{ }^{30}\end{array}$ & $\begin{array}{l}\text { Avaliar o impacto do P4P, associado } \\
\text { ao feedback, sobre a adesão dos } \\
\text { provedores a diretrizes clínicas para o } \\
\text { rastreamento do câncer em mulheres } \\
\text { de } 50 \text { anos de idade e mais. }\end{array}$ & $\begin{array}{l}\text { De } 1993 \text { a 1995, as taxas de triagem duplicaram (de } \\
24 \% \text { para 50\%), sem diferenças significativas entre os } \\
\text { grupos intervenção e controle. Os incentivos financeiros } \\
\text { e feedback não melhoraram a cobertura do rastreamento } \\
\text { de câncer, segundo diretrizes clínicas, entre mulheres de } \\
50 \text { anos de idade e mais. }\end{array}$ & $\begin{array}{l}1993 \text { a } \\
1995\end{array}$ \\
\hline $\begin{array}{l}\text { An et } \\
\mathrm{al}^{31}\end{array}$ & $\begin{array}{l}\text { Comparar o tratamento habitual para } \\
\text { o abandono do tabaco com uso do } \\
\text { P4P para incentivar a referência de } \\
\text { pacientes para serviços de Quitiline } \\
\text { Tobacco (serviços telefônicos de } \\
\text { apoio ao abandono do tabaco) entre } \\
\text { clínicos. }\end{array}$ & $\begin{array}{l}\text { O pagamento por desempenho aumentou o } \\
\text { encaminhamento para serviços de Quitline Tobacco, } \\
\text { especialmente entre médicos com história de menor } \\
\text { engajamento em atividades de melhoria da qualidade. }\end{array}$ & $\begin{array}{l}\text { Setembro } \\
\text { de } 2005 \text { a } \\
\text { junho de } \\
2006\end{array}$ \\
\hline $\begin{array}{l}\text { Chung et } \\
\text { al. }^{32}\end{array}$ & $\begin{array}{l}\text { Avaliar os efeitos da frequência do } \\
\text { pagamento em esquemas de P4P } \\
\text { sobre medidas de qualidade dos } \\
\text { cuidados médicos especializados. }\end{array}$ & $\begin{array}{l}\text { Não houve diferença entre os grupos (controle e } \\
\text { intervenção) na pontuação média da qualidade ou em } \\
\text { quantidade total de bônus ganhos. As respostas dos } \\
\text { médicos a programas de P4P que utilizem incentivos } \\
\text { máximos pequenos não diferem quanto à frequência de } \\
\text { pagamento de bônus. }\end{array}$ & 2007 \\
\hline $\begin{array}{l}\text { Basinga } \\
\text { et al. }{ }^{33}\end{array}$ & $\begin{array}{l}\text { Avaliar os efeitos do P4P sobre o uso } \\
\text { e qualidade dos serviços de cuidados } \\
\text { materno-infantil em Ruanda. }\end{array}$ & $\begin{array}{l}\text { Os incentivos financeiros melhoraram tanto o uso } \\
\text { quanto a qualidade de serviços de saúde materno- } \\
\text { infantil. Os maiores efeitos ocorreram nos serviços com } \\
\text { maiores taxas de pagamento e com menor necessidade de } \\
\text { esforços adicionais dos provedores do serviço. }\end{array}$ & $\begin{array}{l}\text { Junho a } \\
\text { outubro } \\
\text { de } 2006\end{array}$ \\
\hline $\begin{array}{l}\text { Biai et } \\
\text { al. }^{34}\end{array}$ & $\begin{array}{l}\text { Testar se a aplicação rigorosa de } \\
\text { um protocolo clínico para a gestão } \\
\text { da malária associada ao P4P para } \\
\text { provedores de saúde reduziu a } \\
\text { mortalidade hospitalar por malária de } \\
\text { crianças menores de } 5 \text { anos. }\end{array}$ & $\begin{array}{l}\text { A mortalidade hospitalar registrada foi de } 5 \% \text { no } \\
\text { grupo de intervenção e } 10 \% \text { no grupo controle. A } \\
\text { mortalidade cumulativa após quatro semanas da alta } \\
\text { também foi menor no grupo de intervenção. A redução } \\
\text { da mortalidade hospitalar foi associada à fiscalização do } \\
\text { uso de protocolo terapêutico padronizado. Os incentivos } \\
\text { financeiros podem ter contribuído para a motivação na } \\
\text { adesão ao protocolo terapêutico padronizado. }\end{array}$ & $\begin{array}{l}\text { Dezembro } \\
\text { de } 2003 \text { a } \\
\text { novembro } \\
\text { de } 2004 .\end{array}$ \\
\hline $\begin{array}{l}\text { Miller et } \\
\text { al. }^{35}\end{array}$ & $\begin{array}{l}\text { Testar o impacto do P4P na redução } \\
\text { da anemia entre escolares na China } \\
\text { rural. }\end{array}$ & $\begin{array}{l}\text { Os incentivos financeiros foram modestamente eficazes } \\
\text { para melhorar desfechos intermediários. As interações } \\
\text { com outros fatores e condições pré-existentes aos } \\
\text { incentivos financeiros ainda precisam ser esclarecidos. }\end{array}$ & 2011 \\
\hline
\end{tabular}

Fonte: Elaboração própria. 
Estudos observacionais sobre os efeitos do P4P na saúde

Dentre os 37 artigos inicialmente recuperados pela busca sensibilizada, foram incluídos 20 estudos observacionais, sendo 04 do tipo caso-controle, 03 séries temporais interrompidas, 08 estudos de coorte (retrospectiva ou prospectiva), 02 estudos longitudinais, 02 estudos transversais (crosssection) e 01 survey. Embora gozem de menor potencial de recomendação da evidência ${ }^{10-12}$, estudos observacionais podem contribuir com elementos relevantes para a discussão sobre a efetividade do $\mathrm{P} 4 \mathrm{P}$ e, nessa revisão, os principais resultados encontrados, a exemplo dos resultados das revisões sistemáticas e dos ensaios clínicos controlados, também refletiram contradições. O que reforça a ideia de que o $\mathrm{P} 4 \mathrm{P}$ não é uma intervenção uniforme, assim como os resultados passíveis de serem observados, os quais são condicionados por vários fatores que incluem especialmente o modelo e a implementação, mas também elementos contextuais que potencialmente afetam os resultados proporcionados pela intervenção.

Uma parte considerável dos artigos incluídos (07) apresentaram efeitos positivos do P4P sobre diversos desfechos de qualidade e acesso a serviços e ações de saúde ${ }^{28,36-41}$, inclusive em nível sistêmico ${ }^{42}$. Por outro lado, pelo menos sete estudos $^{43-49}$, dentre os que foram incluídos nesta revisão, demostraram inexistir diferenças significativas associadas ao $\mathrm{P} 4 \mathrm{P}$ entre os grupos avaliados e outros 06 identificaram efeitos adversos a partir da utilização do pagamento de incentivos associados ao desempenho ${ }^{37,46,49-52}$. Dois estudos, que não avaliaram especificamente a efetividade do P4P para melhorar resultados, abordaram o desenvolvimento de instrumento avaliativo dos resultados do $\mathrm{P}_{4} \mathrm{P}^{53}$ e a adequação dos valores pagos por procedimento ortopédico ao tempo gasto pela equipe cirúrgica ${ }^{54}$.

O Quadro 4 apresenta os principais resultados extraídos dos estudos observacionais incluídos nesta revisão. Os resultados dos estudos observacionais não forneceram suporte para reconhecer a uniformidade da efetividade dos esquemas de $\mathrm{P} 4 \mathrm{P}$ na melhoria da qualidade ou obtenção de padrões no âmbito dos serviços de saúde. Alguns dos estudos apresentaram resultados favoráveis ao $\mathrm{P} 4 \mathrm{P}$ e defenderam a melhoria de resultados nas condições incentivadas, enquanto outros, especialmente os que avaliaram aspectos complexos, como disparidades em saúde e resultados influenciados por contextos institucionais ou socioeconômicos, bem como os que incluíram o exame dos efeitos adver- sos da utilização do P4P, indicaram que resultados indesejáveis podem advir desses esquemas de incentivos, dependendo do modelo e forma de implantação, reforçando assim, as conclusões anteriormente obtidas a partir dos estudos de revisões sistemáticas e ensaios clínicos controlados incluídos nesta revisão de literatura.

\section{Síntese dos resultados \\ e nível de recomendação da evidência}

Para categorização dos resultados dos diversos estudos incluídos nessa revisão, se considerou especialmente o aspecto da efetividade do $\mathrm{P} 4 \mathrm{P}$ em face dos objetivos propostos pelo esquema estudado. Para isso, foram considerados somente os estudos que visaram especificamente a esse tipo de análise, excluindo-se do quadro síntese de resultados aqueles estudos que, embora relevantes para a discussão dos efeitos da intervenção, não avaliaram em alguma medida os resultados obtidos em função do P4P.

As revisões sistemáticas foram consideradas como a evidência de mais alto nível de recomendação $(\boldsymbol{\Delta} \mathbf{\Delta} \boldsymbol{\Delta})$, seguidas dos ensaios clínicos controlados ( $\mathbf{\Delta} \mathbf{\Delta})$, os quais foram considerados como evidência superior aos estudos observacionais ( $\Delta$ ) quanto ao nível de recomendação, seguindo a classificação preconizada em âmbito internacional ${ }^{10-12}$. O Quadro 5 apresenta o panorama geral desta revisão, considerando a efetividade do P4P e o nível de recomendação da evidência.

Percebeu-se que dentre as evidências com mais alto nível de recomendação, as conclusões foram predominantemente conservadoras, no sentido de reconhecer a indisponibilidade de evidências que sustentem a efetividade do P4P para obtenção de melhores resultados na saúde. Por outro lado, muitos estudos também mostraram que os incentivos podem ser eficazes para produzir os resultados objetivados.

Do total de 38 estudos incluídos no Quadro 5 , dentre os 13 estudos que integram o mais alto nível de recomendação (Revisões Sistemáticas), 04 foram favoráveis à efetividade do $\mathrm{P} 4 \mathrm{P}, 01$ contrário e 08 reconheceram a insuficiência de evidências para afirmar a efetividade da intervenção. Dentre os ensaios clínicos controlados, também considerados com estudos com bom nível de recomendação, 04 observaram a efetividade do P4P nas suas conclusões, 02 implicaram a não efetividade e 01 restou inconclusivo. Entre os estudos observacionais, 12 artigos reportaram efeitos decorrentes da utilização do $\mathrm{P} 4 \mathrm{P}$ e 06 a indiferença dos resultados observados para com a intervenção. 
Quadro 4. Síntese dos resultados da revisão da literatura (Estudos observacionais).

\begin{tabular}{|c|c|c|c|}
\hline \multicolumn{4}{|c|}{ Estudos observacionais (20 estudos incluídos) } \\
\hline Estudo & Objetivos do estudo & Principais resultados de interesse para o $\mathrm{P} 4 \mathrm{P}$ & Período \\
\hline $\begin{array}{l}\text { Huntington } \\
\text { et al. }{ }^{42}\end{array}$ & $\begin{array}{l}\text { Caso-controle. Investigar o } \\
\text { efeito do P4P sobre a entrega } \\
\text { de um pacote básico de serviços } \\
\text { de saúde materno-infantil em } \\
\text { unidades básicas de saúde, no } \\
\text { Egito. }\end{array}$ & $\begin{array}{l}\text { Houve melhoria significativa na qualidade do } \\
\text { planejamento familiar, cuidados pré-natais e } \\
\text { serviços de creche, segundo relato das mulheres } \\
\text { atendidas nas unidades de saúde onde o regime } \\
\text { de P4P estava implantado. Não foram observadas } \\
\text { diferenças significativas nas características } \\
\text { dos prestadores de serviços e clientes, assim } \\
\text { como nas instalações utilizadas pelos grupos } \\
\text { de estudo. Outros fatores, não identificados } \\
\text { pelo estudo, também podem ter influenciado o } \\
\text { comportamento dos prestadores de serviços. }\end{array}$ & $\begin{array}{l}\text { Janeiro a } \\
\text { novembro } \\
\text { de } 2006\end{array}$ \\
\hline $\begin{array}{l}\text { Beaulieu e } \\
\text { Horrigan }^{36}\end{array}$ & $\begin{array}{l}\text { Coorte. Investigar os efeitos } \\
\text { do P4P endereçado a médicos } \\
\text { sobre medidas de qualidade } \\
\text { dos cuidados com o diabetes, } \\
\text { combinados com outras } \\
\text { ferramentas da gestão da } \\
\text { assistência. }\end{array}$ & $\begin{array}{l}\text { Médicos e pacientes conseguiram melhoria } \\
\text { significativa em cinco das seis medidas de } \\
\text { qualidade do cuidado. Os médicos também } \\
\text { melhoraram sua capacidade de ganhar a } \\
\text { recompensa financeira. Os incentivos financeiros } \\
\text { levaram a uma melhoria na qualidade dos } \\
\text { cuidados para os pacientes diabéticos no grupo } \\
\text { estudado, entretanto, pequeno tamanho da } \\
\text { amostra limita a generalização dos resultados. }\end{array}$ & $\begin{array}{l}\text { Abril de } \\
2001 \text { a } \\
\text { janeiro de } \\
2002\end{array}$ \\
\hline Lee et al. $^{43}$ & $\begin{array}{l}\text { Séries temporais interrompidas } \\
\text { (coorte). Analisar o impacto } \\
\text { do Quality and Outcomes } \\
\text { Framework (QOF) (principal } \\
\text { programas de P4P do Reino } \\
\text { Unido) sobre as disparidades } \\
\text { em saúde nas doenças } \\
\text { hipertensivas e coronarianas } \\
\text { entre diferentes grupos étnicos. }\end{array}$ & $\begin{array}{l}\text { O QOF resultou em significativas melhorias } \\
\text { de curto prazo no controle da pressão arterial. } \\
\text { A magnitude do benefício ainda varia entre } \\
\text { grupos étnicos e, de forma geral, as disparidades } \\
\text { no controle dos fatores de risco foram pouco } \\
\text { atenuadas pelo P4P. }\end{array}$ & $\begin{array}{l}2000 \mathrm{a} \\
2007\end{array}$ \\
\hline $\begin{array}{l}\text { Whang et } \\
\text { al. }^{54}\end{array}$ & $\begin{array}{l}\text { Caso-controle. Determinar } \\
\text { se os valores dos pagamentos } \\
\text { para os procedimentos de } \\
\text { descompressão lombar } \\
\text { isoladamente e para } \\
\text { descompressão combinada } \\
\text { com fusão instrumentada, se } \\
\text { adequam ao tempo dispendido } \\
\text { em cada tipo de operação. }\end{array}$ & $\begin{array}{l}\text { Os procedimentos de descompressão combinada } \\
\text { com fusão instrumentada foram associados com } \\
\text { um maior tempo médio cirúrgico. O pagamento } \\
\text { adicional deste procedimento não constituiu } \\
\text { incentivo financeiro indevido para recomendar } \\
\text { a descompressão lombar combinada com o } \\
\text { procedimento de fusão instrumentada para } \\
\text { pacientes com degeneração lombar sintomática, } \\
\text { considerando o maior tempo dispendido, esforço } \\
\text { e risco dessa cirurgia. }\end{array}$ & 2007 \\
\hline $\begin{array}{l}\text { Chan et } \\
\text { al. }{ }^{44}\end{array}$ & $\begin{array}{l}\text { Coorte. Examinar experiência } \\
\text { americana com programa de } \\
\text { P4P (Medicare's Incentive } \\
\text { Payment, MIP) e determinar } \\
\text { como se deu a utilização dos } \\
\text { bônus financeiros e que tipos de } \\
\text { médicos os receberam. }\end{array}$ & $\begin{array}{l}\text { A coorte incluiu } 39.749 \text { prestadores e mais de } \\
\text { quatro milhões de dólares em bônus foram } \\
\text { distribuídos com provedores de cuidados em } \\
\text { saúde. Especialistas e provedores urbanos } \\
\text { concentraram os bônus reembolsos. Dois } \\
\text { milhões de dólares em pagamentos não foram } \\
\text { distribuídos porque não foram reclamados. Os } \\
\text { resultados do P4P, no âmbito do MIP, foram } \\
\text { pequenos em relação ao seu propósito. }\end{array}$ & 1998 \\
\hline
\end{tabular}




\begin{tabular}{|c|c|c|c|}
\hline \multicolumn{4}{|c|}{ Quadro 4. continuação } \\
\hline \multicolumn{4}{|c|}{ Estudos observacionais (20 estudos incluídos) } \\
\hline Estudo & Objetivos do estudo & Principais resultados de interesse para o $\mathrm{P} 4 \mathrm{P}$ & Período \\
\hline $\begin{array}{l}\text { Forsberg et } \\
\text { al. }{ }^{37}\end{array}$ & $\begin{array}{l}\text { Coorte. Investigar se o P4P } \\
\text { é uma forma efetiva para } \\
\text { incentivar a melhoria dos } \\
\text { cuidados de saúde }\end{array}$ & $\begin{array}{l}\text { O P4P, em comparação com um sistema de } \\
\text { orçamento anual, cria motivação que pode } \\
\text { superar o incentivo externo de pressões } \\
\text { financeiras. Entretanto, também pode levar } \\
\text { a efeitos negativos sobre a qualidade do } \\
\text { atendimento. }\end{array}$ & $\begin{array}{l}1996 \mathrm{a} \\
2000\end{array}$ \\
\hline $\begin{array}{l}\text { Ryan e } \\
\text { Blustein }^{45}\end{array}$ & $\begin{array}{l}\text { Coorte. Determinar os } \\
\text { efeitos do programa de P4P } \\
\text { Massachusetts Medicaid } \\
\text { (MassHealth), sobre a qualidade } \\
\text { dos cuidados da pneumonia e } \\
\text { na prevenção da infecção pós- } \\
\text { cirúrgica. }\end{array}$ & $\begin{array}{l}\text { Os efeitos do programa não foram significativos. } \\
\text { Apesar dos substanciais incentivos financeiros. } \\
\text { O programa de P4P MassHealth não melhorou } \\
\text { a qualidade do cuidado nos primeiros anos de } \\
\text { implementação. }\end{array}$ & $\begin{array}{l}2004 \mathrm{a} \\
2009\end{array}$ \\
\hline $\begin{array}{l}\text { Hamilton } \\
\text { et al. }{ }^{46}\end{array}$ & $\begin{array}{l}\text { Coorte. Avaliar o impacto do } \\
\text { P4P (Quality and Outcomes } \\
\text { Framework, Reino Unido) sobre } \\
\text { os cuidados de saúde primários } \\
\text { e sobre as disparidades na gestão } \\
\text { de diabetes entre idade, sexo e } \\
\text { grupos socioeconômicos. }\end{array}$ & $\begin{array}{l}\text { Nas disparidades por sexo, na gestão fator de } \\
\text { risco, verificou-se uma aproximação entre } \\
\text { homens e mulheres. Quanto à idade, pacientes } \\
\text { mais jovens ( }<45 \text { anos) com diabetes parecem } \\
\text { ter se beneficiado menos do programa do que } \\
\text { os mais velhos, aumentando as disparidades } \\
\text { entres os grupos etários. Pacientes vivendo } \\
\text { em áreas ricas e pobres parecem ter benefícios } \\
\text { semelhantes. }\end{array}$ & 2004 \\
\hline $\begin{array}{l}\text { Forsberg et } \\
\text { al. }{ }^{50}\end{array}$ & $\begin{array}{l}\text { Coorte. Investigar se o P4P } \\
\text { afeta a autonomia profissional } \\
\text { de médicos e se isso traz } \\
\text { consequências para a qualidade } \\
\text { do atendimento, em Estocolmo. }\end{array}$ & $\begin{array}{l}\text { O poder profissional e a autonomia foram } \\
\text { limitados e diminuídos durante o período } \\
\text { estudado. A autonomia profissional foi } \\
\text { considerada determinante para a qualidade do } \\
\text { atendimento clínico. Sugere-se que o P4P não } \\
\text { é efetivo para alcançar os resultados desejados, } \\
\text { devido ao impacto negativo sobre o poder e a } \\
\text { autonomia profissionais dos médicos. }\end{array}$ & $\begin{array}{l}1994 \text { a } \\
1998\end{array}$ \\
\hline $\begin{array}{l}\text { Sanada et } \\
\text { al. }^{38}\end{array}$ & $\begin{array}{l}\text { Caso-controle. Avaliar a eficácia } \\
\text { e o custo-efetividade de um } \\
\text { sistema de P4P para melhorar a } \\
\text { gestão do cuidado de úlceras de } \\
\text { pressão. }\end{array}$ & $\begin{array}{l}\text { O grupo intervenção apresentou taxa de } \\
\text { cicatrização significativamente maior em } \\
\text { comparação com o grupo controle. O P4P foi } \\
\text { associado também com a maior rapidez na cura. } \\
\text { A análise de impacto orçamentário demonstrou } \\
\text { que a introdução deste sistema poderia reduzir os } \\
\text { custos do tratamento de úlceras de pressão graves. }\end{array}$ & 2009 \\
\hline $\begin{array}{l}\text { Meterko et } \\
\text { al. }{ }^{53}\end{array}$ & $\begin{array}{l}\text { Desenvolver um instrumento } \\
\text { para avaliar as atitudes dos } \\
\text { médicos em programas de } \\
\text { incentivo de qualidade e avaliar } \\
\text { sua confiabilidade e validade. }\end{array}$ & $\begin{array}{l}\text { É possível identificar e medir as principais } \\
\text { características de programas de P4P com um } \\
\text { instrumento válido e confiável. Este instrumento } \\
\text { pode ser usado para compreender melhor o } \\
\text { impacto do P4P sobre o comportamento do } \\
\text { médico. }\end{array}$ & $\begin{array}{l}\text { Maio a } \\
\text { dezembro } \\
\text { de } 2004\end{array}$ \\
\hline
\end{tabular}




\begin{tabular}{|c|c|c|c|}
\hline \multicolumn{4}{|c|}{ Quadro 4. continuação } \\
\hline \multicolumn{4}{|c|}{ Estudos observacionais (20 estudos incluídos) } \\
\hline Estudo & Objetivos do estudo & Principais resultados de interesse para o $\mathrm{P} 4 \mathrm{P}$ & Período \\
\hline $\begin{array}{l}\text { Millett et } \\
\text { al. } .^{28}\end{array}$ & $\begin{array}{l}\text { Séries temporais interrompidas } \\
\text { (coorte). Analisar o impacto } \\
\text { do Quality and Outcomes } \\
\text { Framework (QOF) (Reino } \\
\text { Unido) sobre a qualidade } \\
\text { dos cuidados para pacientes } \\
\text { de diabetes com e sem } \\
\text { comorbidades. }\end{array}$ & $\begin{array}{l}\text { Melhorias foram evidentes nos dois grupos } \\
\text { acompanhados. Entretanto, pacientes com } \\
\text { comorbidade parecem ter se beneficiado mais } \\
\text { dos efeitos do P4P. }\end{array}$ & $\begin{array}{l}2004 \mathrm{a} \\
2005\end{array}$ \\
\hline $\begin{array}{l}\text { Alshamsan } \\
\text { et al. } .^{47}\end{array}$ & $\begin{array}{l}\text { Séries temporais interrompidas } \\
\text { (coorte). Examinar os efeitos } \\
\text { de longo prazo do programa } \\
\text { de P4P Quality and Outcomes } \\
\text { Framework (QOF) (Reino } \\
\text { Unido) sobre as disparidades } \\
\text { étnicas nos resultados dos } \\
\text { cuidados do diabetes. }\end{array}$ & $\begin{array}{l}\text { O regime de } \mathrm{P} 4 \mathrm{P} \text { do Reino Unido não reduziu as } \\
\text { disparidades étnicas importantes nos resultados } \\
\text { dos cuidados de doenças crônicas ao longo do } \\
\text { tempo. }\end{array}$ & $\begin{array}{l}2004 \mathrm{a} \\
2005\end{array}$ \\
\hline $\begin{array}{l}\text { Hong e } \\
\text { Linn }^{48}\end{array}$ & $\begin{array}{l}\text { Coorte. Esclarecer a influência } \\
\text { do aumento do valor pago ao } \\
\text { parto vaginal pelo Ministério da } \\
\text { Saúde de Taiwan sobre as taxas } \\
\text { de cesarianas no país. }\end{array}$ & $\begin{array}{l}\text { Os resultados sugerem que o incentivo financeiro } \\
\text { não foi relevante para a redução das cesarianas. }\end{array}$ & $\begin{array}{l}2003 \text { a } \\
2007\end{array}$ \\
\hline $\begin{array}{l}\text { Fleetcroft et } \\
\text { al. } .^{39}\end{array}$ & $\begin{array}{l}\text { Cross-section. Estimar o } \\
\text { potencial de redução na } \\
\text { mortalidade da população em } \\
\text { face do P4P na Inglaterra }\end{array}$ & $\begin{array}{l}\text { O contrato de P4P, potencialmente, reduziu a } \\
\text { mortalidade em } 11 \text { vidas por } 100.000 \text { habitantes, } \\
\text { ao ano. O P4P pode ter proporcionado ganho } \\
\text { substancial de saúde, mas esse benefício foi } \\
\text { limitado por metas de desempenho definidas } \\
\text { abaixo do desempenho básico típico. }\end{array}$ & $\begin{array}{l}2004 \mathrm{a} \\
2006\end{array}$ \\
\hline $\begin{array}{l}\text { Doran et } \\
\text { al. } .^{40}\end{array}$ & $\begin{array}{l}\text { Estudo longitudinal. Descrever } \\
\text { o desempenho das clínicas } \\
\text { de pequeno porte no âmbito } \\
\text { programa de P4P Quality and } \\
\text { Outcomes Framework (Reino } \\
\text { Unido) }\end{array}$ & $\begin{array}{l}\text { No primeiro ano do P4P, as clínicas de menor } \\
\text { porte (menos de } 2.000 \text { pacientes) obtiveram a } \\
\text { consecução de metas clínicas para } 83,8 \% \text { dos } \\
\text { pacientes. O desempenho geral melhorou para } \\
\text { as clínicas de todos os portes ao longo do tempo, } \\
\text { mas, nas de pequeno porte, melhorou mais } \\
\text { rapidamente. O efeito do regime de pagamento } \\
\text { por desempenho parece ter sido útil para reduzir } \\
\text { a variação no desempenho, e para reduzir a } \\
\text { diferença entre clínicas de maior e menor porte. }\end{array}$ & $\begin{array}{l}2004 \mathrm{a} \\
2005 \mathrm{e} \\
2006 \mathrm{a} \\
2007\end{array}$ \\
\hline
\end{tabular}

\section{Considerações finais}

$\mathrm{Na}$ revisão e análise da literatura publicada observou-se que, entre os estudos metodologicamente mais rigorosos, as conclusões favoráveis ao $\mathrm{P} 4 \mathrm{P}$ se mostraram menos frequentes, enquanto os estudos observacionais foram mais favoráveis à observação de efeitos positivos do $\mathrm{P} 4 \mathrm{P}$ sobre a qualidade e acesso a serviços de saúde. Limitações metodológicas dos estudos observacionais, com a possibilidade de vieses ou influência de fatores não controlados nas análises, podem ter contribuído para tais resultados, mas, provavelmente, a variedade de resultados encontrados 


\begin{tabular}{|c|c|c|c|}
\hline \multicolumn{4}{|c|}{ Quadro 4. continuação } \\
\hline \multicolumn{4}{|c|}{ Estudos observacionais (20 estudos incluídos) } \\
\hline Estudo & Objetivos do estudo & Principais resultados de interesse para o $\mathrm{P} 4 \mathrm{P}$ & Período \\
\hline $\begin{array}{l}\text { Lester et } \\
\text { al. }^{51}\end{array}$ & $\begin{array}{l}\text { Estudo longitudinal. Avaliar } \\
\text { os efeitos do P4P sobre quatro } \\
\text { indicadores de qualidade } \\
\text { comuns aos programas de P4P } \\
\text { de do Reino Unido e Kaiser } \\
\text { Permanente, Califórnia. }\end{array}$ & $\begin{array}{l}\text { Incentivos para dois indicadores de triagem } \\
\text { (retinopatia diabética e câncer do colo do útero) } \\
\text { foram retirados e repostos durante o período do } \\
\text { estudo, provocando variações no desempenho } \\
\text { relacionado a esses procedimentos. A retirada } \\
\text { dos incentivos financeiros foi associada com uma } \\
\text { diminuição no desempenho de cerca de } 3 \% \text { ao } \\
\text { ano, em média, para a triagem de retinopatia } \\
\text { diabética e cerca de } 1,6 \% \text { ao ano para o rastreio } \\
\text { do câncer do colo do útero. A retirada de } \\
\text { incentivos financeiros a partir de indicadores } \\
\text { clínicos pode provocar declínio dos níveis de } \\
\text { desempenho. }\end{array}$ & $\begin{array}{l}1997 \text { a } \\
2007\end{array}$ \\
\hline Steel et al. ${ }^{52}$ & $\begin{array}{l}\text { Coorte. Analisar a relação entre } \\
\text { as alterações na qualidade } \\
\text { do atendimento registrado } \\
\text { em quatro doenças crônicas } \\
\text { comuns e o P4P. }\end{array}$ & $\begin{array}{l}\text { A introdução do P4P foi associada com a } \\
\text { melhoria da qualidade do atendimento para as } \\
\text { condições incentivadas. Para as condições não } \\
\text { incentivadas, a qualidade não pareceu melhorar. } \\
\text { Pacientes com condições não incentivadas } \\
\text { podem estar em risco de ter cuidados de } \\
\text { qualidade inferior }\end{array}$ & $\begin{array}{l}2003 \text { e } \\
2005\end{array}$ \\
\hline $\begin{array}{l}\text { Gavagan et } \\
\text { al. }{ }^{49}\end{array}$ & $\begin{array}{l}\text { Caso-controle. Avaliar o efeito } \\
\text { de um programa de P4P para } \\
\text { médicos sobre a qualidade do } \\
\text { atendimento preventivo em } \\
\text { uma rede de centros de saúde, } \\
\text { em Houston (EUA). }\end{array}$ & $\begin{array}{l}\text { Não foram observadas diferenças significativas } \\
\text { entre as clínicas que tiveram e que não tiveram } \\
\text { incentivos. A maioria dos médicos considerou } \\
\text { que os incentivos não foram muito eficazes na } \\
\text { melhoria da qualidade do atendimento. }\end{array}$ & $\begin{array}{l}2001 \mathrm{a} \\
2007\end{array}$ \\
\hline $\begin{array}{l}\text { Fiorentini } \\
\text { et al. }{ }^{41}\end{array}$ & $\begin{array}{l}\text { Cross-section. Avaliar os efeitos } \\
\text { de diferentes programas de P4P } \\
\text { sobre hospitalizações evitáveis } \\
\text { na região de Emilia-Romagna } \\
\text { (Itália). }\end{array}$ & $\begin{array}{l}\text { O P4P pode ter um efeito significativo sobre } \\
\text { indicadores agregados de hospitalizações } \\
\text { evitáveis, mas a avaliação da eficácia do esquema } \\
\text { sobre esse desfecho também dever ter em } \\
\text { conta as subpopulações afetadas por doenças } \\
\text { específicas. }\end{array}$ & 2005 \\
\hline
\end{tabular}

Fonte: Elaboração própria.

está muito mais ligada a aspectos conceituais e contextuais da utilização dos esquemas de P4P avaliados, reforçando a heterogeneidade de modelos e resultados do $\mathrm{P} 4 \mathrm{P}$, que não pode ser considerado como uma intervenção uniforme.

Deve-se, portanto, reconhecer que o P4P como uma ferramenta potencialmente útil para promover a motivação necessária ao alcance de objetivos no âmbito de sistemas de saúde, especialmente em mudanças de curto prazo e sobre ações pontuais e que exijam menos esforço dos provedores de serviços de saúde. Entretanto, os resultados dos estudos que envolveram ações e contextos mais complexos, ainda apontam que o P4P deve ser utilizado com cautela e sua aplicação precedida pelo planejamento rigoroso do modelo a ser implantado, observando-se: o agente-alvo do esquema; objetivos pretendidos e metas a ser buscadas; forma de mensuração dos resultados, para o monitoramento e avaliação frequente dos resultados; modalidade de recompensas, quanto à frequência, valor e nível de incentivo buscado; 
Quadro 5. Síntese dos resultados e nível de recomendação da evidência.

\begin{tabular}{|c|c|c|c|c|}
\hline \multirow[b]{2}{*}{ Estudo } & \multicolumn{4}{|c|}{ Resultados acerca da efetividade do P4P } \\
\hline & Nível de recomendação & Efetivo & Não efetivo & Inconclusivo \\
\hline Stone et al. $2002^{14}$ & $\Delta \mathbf{\Delta} \mathbf{\Delta}$ & $\Delta$ & & \\
\hline Chaix-Couturier et al. $2000^{15}$ & $\Delta \mathbf{\Delta} \mathbf{\Delta}$ & $\Delta$ & & \\
\hline Petersen et al. $2006^{16}$ & $\Delta \mathbf{\Delta} \mathbf{\Delta}$ & $\boldsymbol{\Delta}$ & & \\
\hline
\end{tabular}

\begin{tabular}{|l|l}
\hline Giuffrida et al. $2000^{17}$ & \\
\hline Sturm et al. $2007^{18}$ & \\
\hline Witter et al. $2012^{19}$ & \\
\hline Scott et al. $2011^{20}$ &
\end{tabular}

Van Herck et al. $2010^{21}$

de Bruin et al. $2011^{23}$

Eldridge e Palmer $2009^{24}$

Gillam et al. $2012^{25}$

Oxman e Fretheim $2009^{26}$

$\boldsymbol{\Delta} \mathbf{\Delta}$

$\mathbf{\Delta} \mathbf{\Delta}$

$\mathbf{\Delta} \mathbf{\Delta}$

$\mathbf{\Delta} \mathbf{\Delta}$

$\Delta \mathbf{\Delta} \boldsymbol{\Delta}$

$\Delta \mathbf{\Delta} \Delta$

$\Delta \mathbf{\Delta}$

$\Delta \mathbf{\Delta} \Delta$

Emmert et al. $2012^{27}$

Kouides et al. $1998^{29}$

Hillman et al. $1998^{30}$

An et al. $2008^{31}$

Chung et al. $2010^{32}$

Basinga et al. $2011^{33}$

Biai et al. $2007^{34}$

Miller et al. $2012^{35}$

Huntington et al. 2010 ${ }^{42}$

Beaulieu e Horrigan $2005^{36}$

Lee et al. $2011^{43}$

Chan et al. $2004^{44}$

Forsberg et al. $2001^{37}$

Ryan e Blustein $2011^{45}$

Hamilton et al. $2010^{46}$

Forsberg et al. $2001^{50}$

Sanada et al. $2010^{38}$

Millett et al. $2009^{28}$

Alshamsan et al. 2012 ${ }^{47}$

Hong e Linn $2007^{48}$

Fleetcroft et al. $2010^{39}$

Doran et al. $2010^{40}$

Lester et al. $2010^{51}$

Steel et al. $2007^{52}$

Gavagan et al. 2010 49

Fiorentini et al. 2011 $1^{41}$

$\Delta \Delta$

$\Delta \mathbf{A}$

$\Delta$

$\Delta \mathbf{A}$

$\Delta \mathbf{\Delta}$

$\Delta \mathbf{\Delta}$

$\Delta \mathbf{A}$

$\Delta \mathbf{A}$

$\boldsymbol{\Delta} \mathbf{A}$

$\Delta$

$\triangle$

$\Delta$

$\Delta$

$\Delta$

$\Delta$

$\Delta$

$\Delta$

A

$\Delta$

$\Delta$

$\Delta$

A

$\Delta$

$\Delta$

Fonte: Elaboração própria. 
bem como a consideração dos fatores institucionais e contextuais, os quais podem afetar de forma significativa os resultados obtidos. Por fim, deve-se considerar também a possibilidade do $\mathrm{P} 4 \mathrm{P}$ produzir efeitos não planejados, indesejáveis ou adversos, os quais podem eventualmente superar os benefícios pretendidos.

Para o Brasil, o mapeamento, monitoramento e avaliação criteriosos do P4P na saúde, a exemplo do PMAQ, podem ampliar em escala global o conhecimento sobre possibilidades e limitações, bem como sobre os efeitos do seu uso, no entanto, também é extremamente importante que a gestão local esteja bem informada para desenvolver planejamento criterioso, que inclua as melhores evidências disponíveis, para reduzir riscos potenciais e aumentar as chances de bons resultados. 


\section{Referências}

1. Oxman AD, Fretheim A. Can paying for results help to achieve the Millennium Development Goals? Overview of the effectiveness of results-based financing. J Evid Based Med 2009; 2(2):70-83.

2. Canavan A, Toonen J, Elovainio R. Performance Based Financing: An international review of the literature. KIT Development Policy \& Practice; 2008. [acessado 2013 jan 20]. Disponível em: http://www.kit.nl/net/ KIT_Publicaties_output/ShowFile2.aspx?e $=1533$

3. Savedoff WD. Economics of Results-Based Financing in Health. The World Bank; 2010. [acessado 2013 jan 20]. Disponível em: https://www.rbfhealth.org/rbfhealth/library/doc/557/basic-economics-results-based-financing-health

4. Hasan R, Vermeersch C, Rothenbuhler E. Learning from implementation for results-based Financing programs in health: conceptual framework and methods. The World Bank; 2012. [acessado 2013 jan 28]. Disponível em: https://www.rbfhealth.org/rbfhealth/library/doc/684/ learning-implementation-rbf-programs-health-conceptual-framework-and-methods

5. Mannion R, Davies HTO. Payment for performance in health care. BMJ 2008; 336(7639):306-308.

6. Pearson SD, Schneider EC, Kleinman KP, Coltin KL, Singer A. The impact of pay-for-performance on health care quality in Massachusetts, 2001-2003. Health Aff (Millwood) 2008; 27(4):1167-1176.

7. Brasil. Ministério da Saúde (MS). Programa Nacional de Melhoria do Acesso e Qualidade. Departamento de Atenção Básica da Secretaria de Atenção à Saúde; 2012. [acessado 2012 set 21]. Disponível em: http://dab.saude.gov.br/sistemas/Pmaq/

8. Portela GZ, Robeiro JM. A sustentabilidade econômico-financeira da Estratégia Saúde da Família em municípios de grande porte. Cien Saude Colet 2011; 16(3):1719-1732;

9. Cecilio LCO, Andreazza R, Carapinheiro G, Araújo EC, Oliveira LA, Andrade MGG, Meneses CS, Pinto NRS, Reis DO, Santiago S, Souza ALM, Spedo SM. A Atenção Básica à Saúde e a construção das redes temáticas de saúde: qual pode ser o seu papel? Cien Saude Colet 2012; 17(11): 2893-2902.

10. Guyatt G, Rennie D, Meade OM, Cook DJ. Diretrizes para Utilização da Literatura Médica: Fundamentos para a prática clínica da medicina baseada em evidências. $2^{\mathrm{a}}$ ed. Porto Alegre: Artmed; 2011.

11. Brasil. Ministério da Saúde (MS). Secretaria de Ciência Tecnologia e Insumos Estratégicos. Departamento de Ciência e Tecnologia. Diretrizes Metodológicas: elaboração de pareceres técnico-científicos. $3^{\mathrm{a}}$ ed. revisada e atualizada. Brasília: MS; 2011

12. Brasil. Ministério da Saúde (MS). Secretaria de Ciência Tecnologia e Insumos Estratégicos. Departamento de Ciência e Tecnologia. Diretrizes Metodológicas: elaboração de revisão sistemática e metanálise de ensaios clínicos randomizados. Brasília: MS; 2012.

13. Lavis JN, Oxman AD, Lewin S, Fretheim A. SUPPORT Tools for evidence-informed health Policymaking (STP). Health Res Policy Syst 2009; 7(Supl. 1):I1. [acessado 2012 dez 20]. Disponível em: http://www.health -policy-systems.com/content/pdf/1478-4505-7-S1-I1. pdf
14. Stone EG, Morton SC, Hulscher ME, Maglione MA, Roth EA, Grimshaw JM, Mittman BS, Rubenstein LV, Rubenstein LZ, Shekelle PG. Interventions that increase use of adult immunization and cancer screening services: a meta-analysis. Ann Intern Med 2002; 136(9):641-651.

15. Chaix-Couturier C, Durand-Zaleski I, Jolly D, Durieux P. Effects of financial incentives on medical practice: results from a systematic review of the literature and methodological issues. Int J Qual Health Care 2000; 12(2):133-142.

16. Petersen LA, Woodard LD, Urech T, Daw C, Sookanan S. Does pay-for-performance improve the quality of health care? Ann Intern Med 2006; 145(4):265-272.

17. Giuffrida A, Gosden T, Forland F, Kristiansen IS, Sergison M, Leese B, Pedersen L, Sutton M. Target payments in primary care: effects on professional practice and health care outcomes. Cochrane Database of Systematic Reviews 2000; (3):CD000531.

18. Sturm H, Austvoll-Dahlgren A, Aaserud M, Oxman AD, Ramsay C, Vernby A, Kösters JP. Pharmaceutical policies: effects of financial incentives for prescribers. Cochrane Database of Systematic Reviews 2007; (3):CD006731.

19. Witter S, Fretheim A, Kessy FL, Lindahl AK. Paying for performance to improve the delivery of health interventions in low- and middle-income countries. Cochrane Database of Systematic Reviews 2012; 2:CD007899.

20. Scott A, Sivey P, Ait Ouakrim D, Willenberg L, Naccarella L, Furler J, Young D. The effect of financial incentives on the quality of health care provided by primary care physicians.Cochrane Database of Systematic Reviews 2011; (9):CD008451.

21. Van Herck P, De Smedt D, Annemans L, Remmen R, Rosenthal MB, Sermeus W. Systematic review: Effects, design choices, and context of pay-for-performance in health care. BMC Health Serv Res 2010; 10:247.

22. Lee JY, Lee SI, Jo MW. Lessons from healthcare providers attitudes toward pay-for-performance: what should purchasers consider in designing and implementing a successful program? Prev Med Public Health 2012; 45(3):137-147.

23. de Bruin SR, Baan CA, Struijs JN. Pay-for-performance in disease management: a systematic review of the literature. BMC Health Serv Res 2011; 11:272.

24. Eldridge C, Palmer N. Performance-based payment: some reflections on the discourse, evidence and unanswered questions. Health Policy Plan 2009; 24(3):160166.

25. Gillam JS, Siriwardena AN, Steel N. Pay-for-Performance in the United Kingdom: Impact of the Quality and Outcomes Framework-A Systematic Review. Ann Fam Med 2012; 10(5):461-468.

26. Oxman AD, Fretheim A. Can paying for results help to achieve the Millennium Development Goals? Overview of the effectiveness of results-based financing. J Evid Based Med 2009; 2(2):70-83.

27. Emmert M, Eijkenaar F, Kemter H, Esslinger AS, Schöffski O. Economic evaluation of pay-for-performance in health care: a systematic review. Eur J Health Econ 2012; 13(6):755-767 
28. Millett C, Bottle A, Ng A, Curcin V, Molokhia M, Saxena S, Majeed A. Pay for performance and the quality of diabetes management in individuals with and without co-morbid medical conditions. J R Soc Med 2009; 102(9):369-377.

29. Kouides RW, Bennett NM, Lewis B, Cappuccio JD, Barker WH, LaForce FM. Performance-based physician reimbursement and influenza immunization rates in the elderly. The Primary-Care Physicians of Monroe County. Am J Prev Med 1998; 14(2):89-95.

30. Hillman AL, Ripley K, Goldfarb N, Nuamah I, Weiner J, Lusk E. Physician financial incentives and feedback: failure to increase cancer screening in Medicaid managed care. Am J Public Health 1998; 88(11):1699-1701.

31. An LC, Bluhm JH, Foldes SS, Alesci NL, Klatt CM, Center BA, Nersesian WS, Larson ME, Ahluwalia JS, Manley MW. A randomized trial of a pay-for-performance program targeting clinician referral to a state tobacco quitline. Arch Intern Med 2008; 168(18):1993-1999.

32. Chung S, Palaniappan L, Wong E, Rubin H, Luft H Does the frequency of pay-for-performance payment matter? Experience from a randomized trial. Health Serv Res 2010; 45(2):553-564.

33. Basinga P, Gertler PJ, Binagwaho A, Soucat AL, Sturdy J, Vermeersch CM. Effect on maternal and child health services in Rwanda of payment to primary health-care providers for performance: an impact evaluation. Lancet 2011; 377(9775):1421-1428

34. Biai S, Rodrigues A, Gomes M, Ribeiro I, Sodemann M, Alves F, Aaby P. Reduced in-hospital mortality after improved management of children under 5 years admitted to hospital with malaria: randomised trial. $B M$ J 2007; 335(7625):862.

35. Miller G, Luo R, Zhang L, Sylvia S, Shi Y, Foo P, Zhao Q, Martorell R, Medina A, Rozelle S. Effectiveness of provider incentives for anaemia reduction in rural China: a cluster randomised trial. BMJ 2012; 345:e4809.

36. Beaulieu ND, Horrigan DR. Putting smart money to work for quality improvement. Health Serv Res 2005; 40(5 Pt 1):1318-1334.

37. Forsberg E, Axelsson R, Arnetz B. Financial incentives in health care. The impact of performance-based reimbursement. Health Policy 2001; 58(3):243-262.

38. Sanada H, Nakagami G, Mizokami Y, Minami Y, Yamamoto A, Oe M, Kaitani T, Iizaka S. Evaluating the effect of the new incentive system for high-risk pressure ulcer patients on wound healing and cost-effectiveness: a cohort study. Int J Nurs Stud 2010; 47(3):279-286.

39. Fleetcroft R, Parekh-Bhurke S, Howe A, Cookson R, Swift L, Steel N. The UK pay-for-performance programme in primary care: estimation of population mortality reduction. Br J Gen Pract 2010; 60(578): e345-e352.

40. Doran T, Campbell S, Fullwood C, Kontopantelis E, Roland M. Performance of small general practices under the UK's Quality and Outcomes Framework. $\mathrm{Br}$ J Gen Pract 2010; 60(578):e335-344.

41. Fiorentini G, Iezzi E, Lippi Bruni M, Ugolini C. Incentives in primary care and their impacton potentially avoidable hospital admissions. Eur J Health Econ 2011; 12(4):297-309.

42. Huntington D, Zaky HH, Shawky S, Fattah FA, El-Hadary E. Impact of a service provider incentive payment scheme on quality of reproductive and child-health services in Egypt. J Health Popul Nutr 2010; 28(3):273280
43. Lee JT, Netuveli G, Majeed A, Millett C. The effects of pay for performance on disparities in stroke, hypertension, and coronary heart disease management: interrupted time series study. PLoS One 2011; 6(12):e27236.

44. Chan L, Hart LG, Ricketts TC 3rd, Beaver SK. An analysis of Medicare's Incentive Payment program for physicians in health professional shortage areas. J Rural Health 2004; 20(2):109-117.

45. Ryan AM, Blustein J. The effect of the Mass Health hospital pay-for-performance program on quality. Health Serv Res 2011; 46(3):712-728.

46. Hamilton FL, Bottle A, Vamos EP, Curcin V, Anthea, Molokhia M, Majeed A, Millett C. Impact of a pay-for-performance incentive scheme on age, sex, and socioeconomic disparities in diabetes management in UK primary care. J Ambul Care Manage 2010; 33(4):336-349.

47. Alshamsan R, Lee JT, Majeed A, Netuveli G, Millett C. Effect of a UK pay-for-performance program on ethnic disparities in diabetes outcomes: interrupted time series analysis. Ann Fam Med 2012; 10(3):228-234

48. Hong YC, Linn GC. Financial incentives and use of Cesarean delivery: Taiwan birth data 2003 to 2007. Am J Manag Care 2012; 18(1):e35-41.

49. Gavagan TF, Du H, Saver BG, Adams GJ, Graham DM, McCray R, Goodrick GK. Effect of Financial Incentives on Improvement in Medical Quality Indicators for Primary Care. J Am Board Fam Med 2010; 23(5):622-631.

50. Forsberg E, Axelsson R, Arnetz B. Effects of performance-based reimbursement on the professional autonomy and power of physicians and the quality of care. Int J Health Plann Manage 2001; 16(4):297-310.

51. Lester H, Schmittdiel J, Selby J, Fireman B, Campbell S, Lee J, Whippy A, Madvig P. The impact of removing financial incentives from clinical quality indicators: longitudinal analysis of four Kaiser Permanente indicators. BMJ 2010; 340:c1898.

52. Steel N, Maisey S, Clark A, Fleetcroft R, Howe A. Quality of clinical primary care and targeted incentive payments: an observational study. Br J Gen Pract 2007; 57(539):449-454.

53. Meterko M, Young GJ, White B, Bokhour BG, Burgess JF Junior, Berlowitz D, Guldin MR, Nealon Seibert M. Provider attitudes toward pay-for-performance programs: development and validation of a measurement instrument. Health Serv Res 2006; 41(5):1959-1978. Whang PG, Lim MR, Sasso RC, Skelton A, Brown ZB,

54. Greg AD, Albert TJ, Hilibrand AS, Vaccaro AR. Financial incentives for lumbar surgery: a critical analysis of physician reimbursement for decompression and fusion procedures. J Spinal Disord Tech 2008; 21(6):381386.

Artigo apresentado em 13/03/2014

Aprovado em 17/06/2014

Versão final apresentada em 19/06/2014 